\title{
Erratum to: Long-Period Oscillations of Solar Facular Knots
}

\author{
A. A. Solov'ev ${ }^{a, b}, *$, V. V. Smirnova ${ }^{c}$, and P. V. Strekalova ${ }^{a}$ \\ ${ }^{a}$ Main (Pulkovo) Astronomical Observatory, Russian Academy of Sciences, St. Petersburg, 196140 Russia \\ ${ }^{b}$ Kalmyk State University, Elista, 358000 Russia \\ ${ }^{c}$ Crimean Astrophysical Observatory, Russian Academy of Sciences, Nauchnyi, Crimea, 298409 Russia \\ *e-mail:solov.a.a@mail.ru \\ Received September 1, 2020; revised September 2, 2020; accepted September 2, 2020
}

DOI: $10.1134 / \mathrm{S} 1990341320040057$

The original paper can be found online at https://doi.org/10.1134/S1990341320020133. The affiliation of V. V. Smirnova in the original paper was erroneous and need to be corrected. The correct affiliation is:

Crimean Astrophysical Observatory, Russian Academy of Sciences, Nauchnyi, Crimea, 298409 Russia 